\title{
Correction
}

\section{Correction to: Weighted Energy-Dissipation approach to doubly nonlinear problems on the half line}

\author{
Goro Akagi(i), Stefano Melchionna And Ulisse Stefanelli
}

\section{Correction to: J. Evol. Equ. 18 (2018), 49-74}

\section{https://doi.org/10.1007/s00028-017-0390-6}

In our paper [2], strong solutions of the Cauchy problem for the doubly nonlinear gradient flow posed on the dual space $V^{*}$ of a uniformly convex Banach space $V$,

$$
\begin{aligned}
\xi(t)+\eta(t) & =0, \quad \xi(t)=\mathrm{d}_{V} \psi\left(u^{\prime}(t)\right), \quad \eta(t) \in \partial \phi(u(t)) \text { in } V^{*} \text { for a.e. } t>0, \\
u(0) & =u_{0}
\end{aligned}
$$

where $\mathrm{d}_{V} \psi: V \rightarrow V^{*}$ (resp. $\partial \phi: V \rightarrow 2^{V^{*}}$ ) denotes the Gâteaux derivative (resp. subdifferential) of a smooth (resp. lower semicontinuous) convex functional defined on $V$, are recovered as a limit as $\varepsilon \rightarrow 0+$ of minimizers

$$
\begin{aligned}
u_{\varepsilon} & \in K\left(u_{0}\right) \\
& :=\left\{u \in W^{1, p}\left(\mathbb{R}_{+}, \mathrm{e}^{-t / \varepsilon} \mathrm{d} t ; V\right): u(0)=u_{0} \text { and } \phi(u) \in L^{1}\left(\mathbb{R}_{+}, \mathrm{e}^{-t / \varepsilon} \mathrm{d} t\right)\right\}
\end{aligned}
$$

of the WED functional

$$
I_{\varepsilon}(u)=\left\{\begin{array}{cc}
\int_{0}^{\infty} \mathrm{e}^{-t / \varepsilon}\left(\varepsilon \psi\left(u^{\prime}(t)\right)+\phi(u(t))\right) \mathrm{d} t & \text { if } u \in K\left(u_{0}\right), \\
+\infty & \text { otherwise. }
\end{array}\right.
$$


In [2], we make the following assumptions with $1<p<+\infty$ :

$$
\begin{aligned}
& |u|_{V}^{p} \leq C(\psi(u)+1) \forall u \in V, \psi(0)=0 ; \\
& \left|\mathrm{d}_{V} \psi(u)\right|_{V^{*}}^{p^{\prime}} \leq C\left(|u|_{V}^{p}+1\right) \forall u \in V, p^{\prime}=p /(p-1) ; \\
& D(\phi) \subset X ; \text { for each } c \text { the set }\{u \in X: \phi(u) \leq c\} \text { is bounded in } X ; \\
& |\eta|_{X^{*}} \leq \ell\left(|u|_{V}+\phi(u)\right) \forall \eta \in \partial_{X} \phi_{X}(u),
\end{aligned}
$$

where $X$ is a reflexive Banach space densely and compactly embedded into $V, \partial_{X} \phi_{X}$ : $X \rightarrow 2^{X^{*}}$ denotes the subdifferential operator of the restriction $\phi_{X}$ of $\phi$ onto $X$ and $\ell(\cdot)$ is a non-decreasing function. The proof in [2] relies on a regularization of $I_{\varepsilon}$ and hinges on the following uniform estimate:

$$
\sup _{t \in[0, \infty)} \phi_{\lambda}\left(u_{\varepsilon \lambda}(t)\right) \leq C
$$

for minimizers $u_{\varepsilon \lambda}$ of regularized WED functionals $I_{\varepsilon \lambda}$, which is given by (3) and (4) with $\phi$ replaced by the Moreau-Yosida regularization $\phi_{\lambda}: V \rightarrow \mathbb{R}$ of $\phi$. The proof of this bound in [2] is however flawed, as pointed out by Mr. Thomas Ruf, whose feedback we gratefully acknowledge. On the other hand, for any $T>0$, one can derive a uniform estimate

$$
\int_{0}^{T} \phi_{\lambda}\left(u_{\varepsilon \lambda}(t)\right) \mathrm{d} t \leq C_{T}
$$

for some constant $C_{T}>0$ independent of $\varepsilon, \lambda \in(0,1)$. Indeed, for any $n \in \mathbb{N}$, it holds that

$$
\int_{\varepsilon n}^{\varepsilon(n+1)} \phi_{\lambda}\left(u_{\varepsilon \lambda}(t)\right) \mathrm{d} t \leq \varepsilon \operatorname{e} \phi\left(u_{0}\right)
$$

(see p. 60 of [2] with the choice $\tau=n$ ). Let $N_{\varepsilon} \in \mathbb{N}$ be such that $\varepsilon N_{\varepsilon} \leq T<\varepsilon\left(N_{\varepsilon}+1\right)$. It then follows that

$$
\int_{0}^{T} \phi_{\lambda}\left(u_{\varepsilon \lambda}(t)\right) \mathrm{d} t \leq \sum_{n=0}^{N_{\varepsilon}} \int_{\varepsilon n}^{\varepsilon(n+1)} \phi_{\lambda}\left(u_{\varepsilon \lambda}(t)\right) \mathrm{d} t \leq\left(N_{\varepsilon}+1\right) \varepsilon \operatorname{e} \phi\left(u_{0}\right) \leq(T+1) \operatorname{e} \phi\left(u_{0}\right)
$$

for $\varepsilon \in(0,1)$. Hence, by replacing (7) and (8) by

$$
\begin{aligned}
& D(\phi) \subset X ;|u|_{X}^{m} \leq C(\phi(u)+1) \quad \forall u \in D(\phi) ; \\
& |\eta|_{X^{*}}^{m^{\prime}} \leq \ell\left(|u|_{V}\right)(\phi(u)+1) \forall \eta \in \partial_{X} \phi_{X}(u)
\end{aligned}
$$

for some $1<m<+\infty$ and a non-decreasing function $\ell$ in $\mathbb{R}$, the argument in [2] entails the validity of the following.

Theorem 1. (WED variational approach). Assume (5), (6), (11), and (12). Then:

(i) The WED functional $I_{\varepsilon}$ admits global minimizers $u_{\varepsilon}$ in $K\left(u_{0}\right)$. Additionally, if either $\phi$ or $\psi$ is strictly convex, the minimizer is unique. 
(ii) For every minimizer $u_{\varepsilon}$ of $I_{\varepsilon}$ on $K\left(u_{0}\right)$ there exists $\eta_{\varepsilon} \in \partial_{X} \phi_{X}\left(u_{\varepsilon}\right)$ such that, by letting $\xi_{\varepsilon}=\mathrm{d}_{V} \psi\left(u_{\varepsilon}^{\prime}\right)$, the triple $\left(u_{\varepsilon}, \eta_{\varepsilon}, \xi_{\varepsilon}\right)$ belonging to

$$
\begin{array}{r}
{\left[W^{1, p}\left(\mathbb{R}_{+}, \mathrm{e}^{-t / \varepsilon} \mathrm{d} t ; V\right) \cap L_{\mathrm{loc}}^{m}\left(\mathbb{R}_{+} ; X\right)\right] \times L_{\mathrm{loc}}^{m^{\prime}}\left(\mathbb{R}_{+} ; X^{*}\right)} \\
\times L^{p^{\prime}}\left(\mathbb{R}_{+}, \mathrm{e}^{-t / \varepsilon} \mathrm{d} t ; V^{*}\right)
\end{array}
$$

is a strong solution of the Euler-Lagrange problem

$$
\begin{aligned}
-\varepsilon \xi_{\varepsilon}^{\prime}(t)+\xi_{\varepsilon}(t)+\eta_{\varepsilon}(t) & =0, \eta_{\varepsilon}(t) \in \partial_{X} \phi_{X}\left(u_{\varepsilon}(t)\right) & & \text { in } X^{*}, \text { for a.e. } t>0, \\
\xi_{\varepsilon}(t) & =\mathrm{d}_{V} \psi\left(u_{\varepsilon}^{\prime}(t)\right) & & \text { in } V^{*}, \text { for a.e. } t>0, \\
u_{\varepsilon}(0) & =u_{0} . & &
\end{aligned}
$$

Moreover, minimizers of $I_{\varepsilon}$ in $K\left(u_{0}\right)$ and strong solutions of the Euler-Lagrange system coincide.

(iii) For any sequence $\varepsilon_{k} \rightarrow 0$ there exists a subsequence (denoted by the same letter) such that $\left(u_{\varepsilon_{k}}, \eta_{\varepsilon_{k}}, \xi_{\varepsilon_{k}}\right) \rightarrow(u, \eta, \xi)$ weakly in

$$
\left[W^{1, p}(0, T ; V) \cap L^{m}(0, T ; X)\right] \times L^{m^{\prime}}\left(0, T ; X^{*}\right) \times L^{p^{\prime}}\left(0, T ; V^{*}\right)
$$

for all $T>0$, where $(u, \eta, \xi)$ is a strong solution to the doubly nonlinear problem,

$$
\begin{aligned}
\xi(t)+\eta(t) & =0, \quad \xi(t)=\mathrm{d}_{V} \psi\left(u^{\prime}(t)\right), \quad \eta(t) \in \partial \phi(u(t)) \text { in } V^{*} \text { for a.e. } t>0, \\
u(0) & =u_{0} .
\end{aligned}
$$

Indeed, combining (10) with (11) and (12), we infer that

$$
\int_{0}^{T}\left|J_{\lambda} u_{\varepsilon \lambda}(t)\right|_{X}^{m} \mathrm{~d} t+\int_{0}^{T}\left|\eta_{\varepsilon \lambda}(t)\right|_{X^{*}}^{m^{\prime}} \mathrm{d} t \leq C_{T},
$$

where $J_{\lambda}: V \rightarrow D(\partial \phi)$ is the resolvent of $\partial \phi$, uniformly for $\varepsilon, \lambda \in(0,1)$. Hence, instead of the corresponding weak convergences in $L^{\rho}(0, T ; X)$ and $L^{\rho}\left(0, T ; X^{*}\right)$ for any $\rho \in(1,+\infty)$ in [2], we use only

$$
\begin{aligned}
J_{\lambda} u_{\varepsilon \lambda} & \rightarrow u_{\varepsilon} \quad \text { weakly in } L^{m}(0, T ; X), \\
\eta_{\varepsilon \lambda} & \rightarrow \eta_{\varepsilon} \quad \text { weakly in } L^{m^{\prime}}\left(0, T ; X^{*}\right)
\end{aligned}
$$

as $\lambda \rightarrow 0+$ for any $T>0$. The weak convergences (16)-(17) are sufficient to identify the limits, e.g., $\eta_{\varepsilon}$, of nonlinear terms (see [1], where the bounded-interval case is treated). The causal limit of $u_{\varepsilon}$ as $\varepsilon \rightarrow 0+$ can be also discussed with the aid of (16) and (17).

By replacing (7)-(8) by (11)-(12), the WED variational approach is no longer applicable to discuss the Kirchhoff-type integrodifferential equation (5.5)-(5.7) of [2] in full generality. However, one can treat the case of a homogeneous $\alpha$, say $\alpha(s)=$ 
$|s|^{p-2} s$ for $1<p<+\infty$. Indeed, one can incorporate the nonlocality $\|\nabla u(t)\|_{2}$ into $\alpha\left(u_{t}\right)$ by making use of the homogeneity of $\alpha$ and a change of the time-variable. To this end, set

$$
u(x, t)=v(x, s), \quad s=\int_{0}^{t}\|\nabla u(\tau)\|_{2}^{-1 /(p-1)} d \tau .
$$

Here, one has to avoid the case $\left\|\nabla u_{0}\right\|_{2}=0$, for which only the trivial solution exists. Then $u_{t}(x, t)=\|\nabla u(t)\|_{2}^{-1 /(p-1)} v_{s}(x, s)$ and $\Delta u(x, t)=\Delta v(x, s)$, and hence, the equation is reduced to

$$
\alpha\left(v_{s}\right)=\Delta v \text { in } \Omega \times \mathbb{R}_{+},
$$

which falls within the scope of Theorem 1.

Publisher's Note Springer Nature remains neutral with regard to jurisdictional claims in published maps and institutional affiliations.

\section{REFERENCES}

[1] G. Akagi, U. Stefanelli, Weighted energy-dissipation functionals for doubly nonlinear evolution, J. Funct. Anal. 260 (2011), 2541-2578.

[2] G. Akagi, S. Melchionna, U. Stefanelli, Weighted energy-dissipation approach to doubly nonlinear problems on the half line, J. Evol. Equ. 18 (2018), 49-74. 
Goro Akagi

Mathematical Institute

Tohoku University

Aoba Sendai 980-8578

Japan

E-mail:akagi@m.tohoku.ac.jp

and

Institut für Computational Biology

Helmholtz Zentrum München

Ingolstädter Landstrasse 1

85764 Neuherberg

Germany

and

Zentrum Mathematik

Technische Universität München

Boltzmannstrasse 3

85748 Garching bei München

Germany

Stefano Melchionna and Ulisse Stefanelli

Faculty of Mathematics

University of Vienna

Oskar-Morgenstern-Platz 1

1090Wien

Austria

E-mail: stefano.melchionna@univie.ac.at

URL: http://www.mat.univie.ac.at/ melchionns90/

Ulisse Stefanelli

E-mail: ulisse.stefanelli@univie.ac.at

URL: http://www.mat.univie.ac.at/ stefanelli

Ulisse Stefanelli

Istituto di Matematica Applicata e Tecnologie

Informatiche E. Magenes

v. Ferrata 1

27100 Pavia

Italy 